\title{
Coherent Perfect Absorbers: Time-Reversed Lasers
}

\author{
Y.D. Chong, ${ }^{*}$ Li Ge, Hui Cao, and A. D. Stone \\ Department of Applied Physics, Yale University, New Haven, Connecticut 06520, USA \\ (Received 25 March 2010; revised manuscript received 14 June 2010; published 26 July 2010)
}

\begin{abstract}
We show that an arbitrary body or aggregate can be made perfectly absorbing at discrete frequencies if a precise amount of dissipation is added under specific conditions of coherent monochromatic illumination. This effect arises from the interaction of optical absorption and wave interference and corresponds to moving a zero of the elastic $S$ matrix onto the real wave vector axis. It is thus the time-reversed process of lasing at threshold. The effect is demonstrated in a simple Si slab geometry illuminated in the 500-900 nm range. Coherent perfect absorbers act as linear, absorptive interferometers, which may be useful as detectors, transducers, and switches.
\end{abstract}

DOI: 10.1103/PhysRevLett.105.053901

PACS numbers: 42.25.Bs, 42.25.Hz, 42.55.Ah

A laser is a physical system which, when subjected to an energy flux (pump), self-organizes at a threshold value of the pump to produce narrow-band coherent electromagnetic radiation. In the absence of inhomogeneous broadening and quantum fluctuations, this radiation has zero linewidth. Above the first lasing threshold, lasers are nonlinear systems, but at the first threshold they satisfy a linear wave equation with a negative (amplifying) imaginary part of the refractive index, generated by the population inversion due to the pump [1]. In conventional lasers, the gain medium is confined in resonators with a relatively high quality factor $(Q)$, and the lasing modes are closely related to passive-cavity modes. However, recent demonstrations of random lasers [2,3] have shown that the lasing threshold can be reached and coherent lasing obtained in resonators with no high- $Q$ passive-cavity modes. It can be rigorously shown within semiclassical laser theory that the first lasing mode in any cavity is an eigenvector of the electromagnetic scattering matrix ( $S$ matrix) with an infinite eigenvalue; i.e., lasing occurs when a pole of the $S$ matrix is pulled "up" to the real axis by including gain as a negative imaginary part of the refractive index [4]. This viewpoint suggests the possibility of the time-reversed process of lasing at threshold [5,6]. A specific degree of dissipation ("loss medium") is added to the resonator, corresponding to a positive imaginary refractive index equal in absolute value to that at the lasing threshold. The system is illuminated coherently and monochromatically by the time reverse of the output of a lasing mode, and the incident radiation is perfectly absorbed. We refer to such an optical system as a coherent perfect absorber (CPA).

Coherent perfect absorption is a general and robust phenomenon related to the analytic properties of the $S$ matrix. For simplicity, we consider scattering in one or two dimensions, for which the electric field (in the TM polarization) is a scalar obeying the Helmholtz equation:

$$
\left[\nabla^{2}+n^{2}(\vec{r}) k^{2}\right] \phi(\vec{r})=0 .
$$

Here $k=\omega / c$ (a scalar), $\omega$ is the frequency, and $n=n^{\prime}+$ $\mathrm{in}^{\prime \prime}$ is the complex refractive index, with $n^{\prime \prime}<0$ for gain and $n^{\prime \prime}>0$ for absorption. An "external region," encompassing the resonator, extends from some radius $r_{s}$ to infinity. Here $n=n_{0}$, a constant $\left(n_{0}=1\right.$ for free space), and the field is a combination of incoming waves $\psi_{m}^{\text {in }}$ and outgoing waves $\psi_{m}^{\text {out }}$ :

$$
\phi(\vec{r})=\sum_{m}\left[\alpha_{m} \psi_{m}^{\text {in }}(\vec{r})+\beta_{m} \psi_{m}^{\text {out }}(\vec{r})\right], \quad r>r_{s} .
$$

The $S$ matrix, $\sum_{m^{\prime}} S_{m m^{\prime}}(k) \alpha_{m^{\prime}}=\beta_{m}$, relates the scattering amplitudes. For lossless media, $n^{\prime \prime}=0$ and $S(k)$ is unitary for real $k$. Continuing $k$ into the complex plane, $S(k)$ has a countably infinite set of poles and zeros, symmetrically placed at $\left\{k_{m}^{\mp}=q_{m} \mp i \gamma_{m}\right\}$ with $\gamma_{m}>0$.

When we add gain or dissipation, the zeros and poles of $S(k)$ flow in the complex $k$ plane, but they cannot simply appear or disappear because $\arg [\operatorname{det}(S)]$ always winds by $2 \pi$ around each zero and $-2 \pi$ around each pole. We parameterize the dissipation by the positive value of the imaginary part of the refractive index, $\operatorname{Im}\{n\}=n^{\prime \prime}>0$. Whereas in the threshold lasing problem adding gain $\left(n^{\prime \prime}<\right.$ 0 ) moves the poles up towards the real axis, adding dissipation generates an exactly symmetric downward motion of the zeros. For some value $n_{m}^{\prime \prime}$, the $m$ th zero crosses the real axis at some real $\tilde{k}_{m}$, leading to a perfect absorption resonance at that frequency. We refer to the discrete pairs $\left\{\left(\tilde{k}_{m}, n_{m}^{\prime \prime}\right)\right\}$ as the CPA zeros; they are in one to one correspondence with the lasing thresholds $\left\{\left(\tilde{k}_{m},-n_{m}^{\prime \prime}\right)\right\}$. Radiation incident at each $\tilde{k}_{m}$ will be completely absorbed if it corresponds to the specific eigenvector of the $S$ matrix having eigenvalue zero, which is simply the time reverse of the corresponding lasing mode. For homogeneous bodies, such purely incoming, real- $k$ radiation modes are the complex conjugate of the constant-flux states first proposed by Kapur and Peierls [7] and recently introduced to laser theory by Türeci, Stone, and Collier [8]. We wish to emphasize that the CPA zeros are distinct from absorption resonances of the atomic or molecular medium, which do not rely on the incoming radiation occurring within spe- 
cific modes. The CPA process arises from the interplay of interference and absorption: In the presence of specific amounts of dissipation, there exist interference patterns that trap the incident radiation for an infinite time. If the resonator of the CPA has high $Q$, then even small rates of single-pass absorption can lead to perfect absorption. Hence, media that normally do not absorb radiation well at certain frequencies can be made to do so, as we demonstrate below for $\mathrm{Si}$ (silicon). Finally, we show that absorption can also be reduced by illuminating the CPA with eigenvectors of the $S$ matrix with constructive interference for escape.

Before moving to specific examples, we discuss a general framework for finding CPA zeros based on the " $R$-matrix" theory of Wigner and Eisenbud [9]. In this approach, the $S$ matrix is given by

$$
S(k)=-e^{2 \mathrm{in}_{0} k r_{0}}\left[I-\mathrm{in}_{0} k R(k)\right]^{-1}\left[I+\mathrm{in}_{0} k R(k)\right],
$$

where $r_{0}>r_{s}$ is an arbitrarily chosen boundary far from the origin, and $R(k)$ (the $R$ matrix) takes the form

$$
\begin{gathered}
R_{m m^{\prime}}(k)=\sum_{\alpha \alpha^{\prime}=1}^{\infty} \phi_{\alpha}^{m} F_{\alpha \alpha^{\prime}}^{-1} \phi_{\alpha^{\prime}}^{m^{\prime}}, \\
F_{\alpha \alpha^{\prime}}=\left(k_{\alpha}^{2}-k^{2}\right) \delta_{\alpha \alpha^{\prime}}-i k^{2} \gamma_{\alpha \alpha^{\prime}},
\end{gathered}
$$

where each $\phi_{\alpha}^{m} \in \mathbb{R}$ is a Wigner-Eisenbud basis function evaluated at $r=r_{0}$ and decomposed into the $m$ th channel [10], and $k_{\alpha} \in \mathbb{R}$ is the corresponding eigenvalue. The dissipation matrix $\gamma_{\alpha \alpha^{\prime}}=\int d^{d} r \operatorname{Im}\left(n^{2}\right)(\vec{r}) \phi_{\alpha}(\vec{r}) \phi_{\alpha^{\prime}}(\vec{r})$ is real and positive-definite.

When $Q \gg 1$, each $S$-matrix zero or pole is determined by approximating the $R$ matrix by a single term $\alpha$; in this case the zeros and poles of the $S$ matrix occur at

$$
\left(1+i \gamma_{\alpha \alpha}\right) k^{2} \mp i \varphi_{\alpha} n_{0} k-k_{\alpha}^{2}=0,
$$

where $\varphi_{\alpha}=\sum_{m}\left(\phi_{\alpha}^{m}\right)^{2}>0$. Without dissipation $\left(\gamma_{\alpha \alpha}=\right.$ 0 ), this implies that all zeros have positive imaginary parts, as already noted. Furthermore, zeros cross the real axis exactly when $\gamma_{\alpha \alpha}=\varphi_{\alpha} / k_{\alpha}$, at frequency $k=k_{\alpha}$. In Fig. 1, we show the exact pole motion, for the case of the two-channel CPA (see inset and discussion below), finding excellent agreement with the single-pole $R$-matrix prediction, even though the cavity only has $Q \sim 30$.

The simplest possible CPA is a single port reflector, in which a single channel fiber or waveguide is terminated by a cavity tuned to the correct value of $n^{\prime \prime}$. This device is similar, although not identical, to the "critically coupled fiber-resonator" systems widely studied in integrated optics $[11,12]$. In a CPA, the loss induced in the resonator is completely due to absorption in the loss medium and not due to outcoupling (e.g., bending) loss. This makes the one-port CPA is potentially useful as an on-chip photovoltaic or calorimetric detector or transducer. However, the optical control properties of the CPA are revealed only when there is more than a single input channel of the incident field. We therefore study a simple two-channel

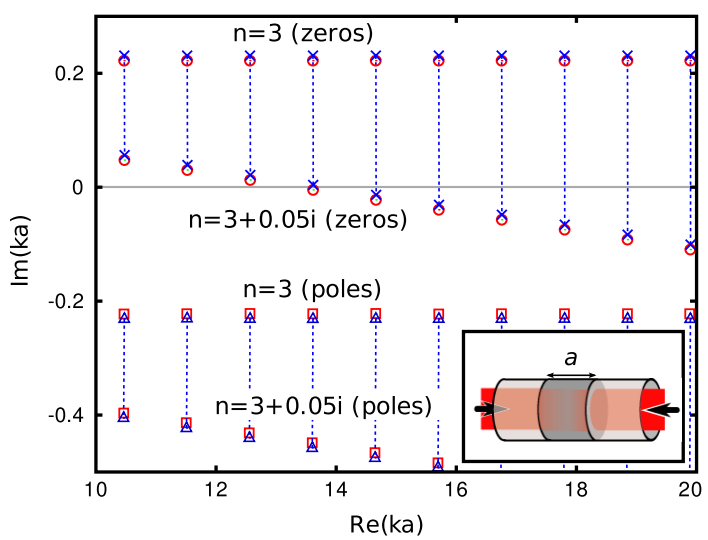

FIG. 1 (color online). Motion of exact $S$-matrix zeros (blue crosses) and poles (blue triangles) in the complex- $k$ plane, as dissipation increases from zero, for a two-channel resonator of length $a$ and uniform index $n$. In the external region, $n_{0}=1$. For $n \approx 3+0.05 i$, the fourth zero from the left touches the real axis, yielding a CPA zero. Also shown are the zeros (red circles) and poles (red squares) predicted from Eq. (6). Inset: Schematic of system; each input is a single-mode fiber.

case to illustrate the concept fully. Consider a single-mode fiber or waveguide with index $n_{0}$ containing a resonator consisting simply of a segment of thickness $a$ and uniform refractive index $n$ (see inset in Fig. 1); in this case, there are two input channels for each propagating $k$, corresponding to incident radiation from the left and right. An almost equivalent system would be a slab of thickness $a$ illuminated on both sides by a narrow beam at normal incidence, in which case $n_{0}=1$. In both cases, it is straightforward to calculate the $2 \times 2 S$ matrix for arbitrary complex index $n$ and find its two eigenvalues $s_{1,2}$. The total scattering intensity for each eigenmode is $\left|s_{1,2}\right|^{2}$, and a zero eigenvalue of $S$ occurs when

$$
e^{i n k a}= \pm \frac{n-n_{0}}{n+n_{0}} .
$$

When $k a \gg 1$, we can find an infinite number of discrete solutions of this equation, $n_{\nu}=n_{\nu}^{\prime}+i n_{\nu}^{\prime \prime}$, as

$$
\begin{gathered}
n_{\nu}^{\prime} \approx \frac{\pi \nu}{n_{0} k a}, \quad \nu=1,2,3, \ldots, \\
n_{\nu}^{\prime \prime} \approx \frac{1}{n_{0} k a} \ln \left(\frac{n_{\nu}^{\prime}+n_{0}}{n_{\nu}^{\prime}-n_{0}}\right) .
\end{gathered}
$$

In Fig. 2, we take $n_{0}=1$ and plot the solutions for $k a=$ 664.7. Note that we have here restated the CPA problem so as to find the $\left\{n_{\nu}(k)\right\}$ which produces a zero of the $S$ matrix for a fixed $k$. This can be achieved by letting both the real and imaginary parts of $n_{\nu}$ vary; earlier, we varied the imaginary part $n^{\prime \prime}$ at fixed $n^{\prime}$, leading to zeros at different $k$ points. This is a useful reformulation because it suggests one practical means to realize a CPA. If the frequencydependent $n(k)$ of the loss medium can be tuned appropriately by scanning $k$, one may achieve the CPA resonance condition, i.e., $n(k) \approx n_{\nu}(k)$ for some integers $\nu$. 


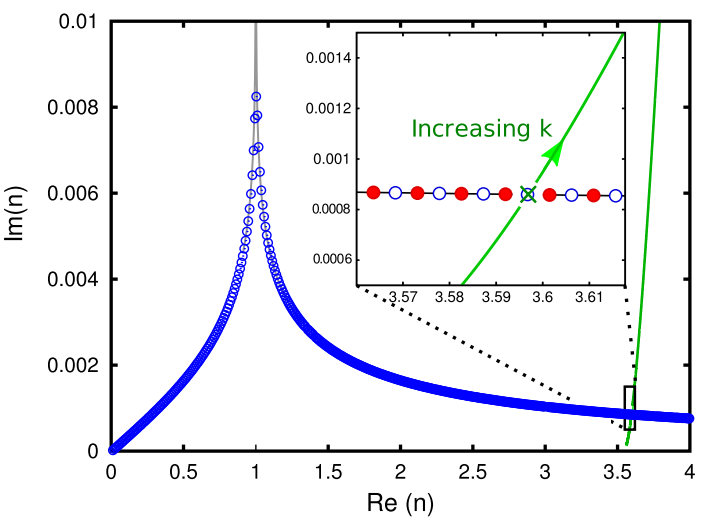

FIG. 2 (color online). Complex refractive indices leading to perfect absorption for the device of Fig. 1, for $k a=664.7$. Only parity-even solutions are shown (blue circles). The green curve shows the refractive index of $\mathrm{Si}, n(k)$. Inset: Parity-even solutions (hollow blue circles) and parity-odd solutions (filled red circles) in the region $3.55<\operatorname{Re}(n)<3.62$; the green cross shows the index of $\mathrm{Si}$ at $k a=664.7, a=100 \mu \mathrm{m}$ (i.e., $\lambda=$ $945.3 \mathrm{~nm})$.

In an indirect band gap semiconductor such as $\mathrm{Si}$ or $\mathrm{GaP}$, $n^{\prime \prime}$ increases smoothly from very small values as $k$ increases past the band gap, while $n^{\prime}$ changes little [13]. For frequencies near the band gap, $\partial n^{\prime \prime} / \partial k \gg \partial n_{\nu}^{\prime \prime} / \partial k$ and $\partial n^{\prime} / \partial k \ll \partial n_{\nu}^{\prime} / \partial k$, and thus it is possible to vary $k$ so as to pass very close to several CPA zeros. Like a bound state, a CPA zero has no intrinsic width; the scattered intensity near a CPA zero is $I_{\min } \sim c_{0}\left|n(k)-n_{\nu}(k)\right|^{2}$, where $c_{0}$ is of order unity. Figure 3 shows the $S$-matrix eigenvalue intensities for a slab of undoped $\mathrm{Si}$, with $a=$ $100 \mu \mathrm{m}$. More than ten substantial CPA minima are visible between 938 and $954 \mathrm{~nm}$, with $\left|n(k)-n_{\nu}(k)\right|^{2} \lesssim 10^{-5}$ and an intensity contrast of up to $\sim 50 \mathrm{~dB}$.

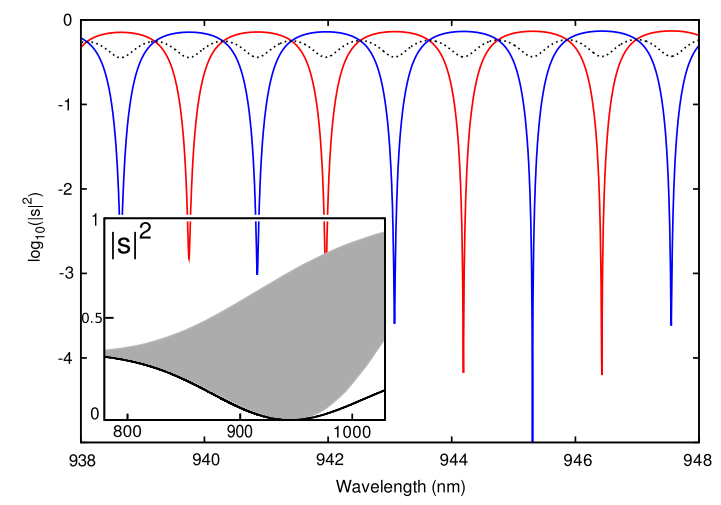

FIG. 3 (color online). Semilog plot of normalized output intensities $|s|^{2}$ vs the wavelength $\lambda=2 \pi / k$, for a $100 \mu \mathrm{m}$ Si slab. Solid lines show $\log _{10}\left(|s|^{2}\right)$ for a parity-even (blue) or parity-odd (red) eigenmode. The dashed line shows $2\left(|r|^{2}+|t|^{2}\right)$, the total output intensity when the two input beams are incoherent. Inset: Upper and lower bounds for $|s|^{2}$ over a wide range of $\lambda$. The gray area shows the actual bounds, and the solid black line show the approximate lower bound from Eq. (10).
A crucial point is that the system exhibits perfect absorption only if it is coherently illuminated with the zero eigenmode of the $S$ matrix for the resonant $k$ value. Because of the mirror symmetry of this uniform index CPA, the two eigenmodes are parity eigenstates, meaning that the left and right beams must have phase difference 0 or $\pi$. The \pm in Eq. (7) determines whether the perfectabsorber condition is satisfied by the symmetric or antisymmetric mode. The location of these minima is $a$-dependent and hence tunable within a given material; we can derive a tight lower bound for the $S$-matrix eigenvalue intensities:

$$
|s(a)|^{2} \geq\left[\frac{2\left(n^{\prime 2}-n_{0}\right) \sinh \left(n^{\prime \prime} k a\right)-4 n^{\prime} n_{0}}{\left(n^{\prime}+n_{0}\right)^{2} e^{n^{\prime \prime} k a}+\left(n^{\prime}-n_{0}\right)^{2} e^{-n^{\prime \prime} k a}}\right]^{2},
$$

which goes to zero at the $n$ values given in (7) and locates the interesting operating regions. For the uniform Si CPA, the optimal wavelength occurs at around $750 \mathrm{~nm}$ for $a=$ $10 \mu \mathrm{m}$ and around $1000 \mathrm{~nm}$ for $a=150 \mu \mathrm{m}$; the exact value depends on $n(k)$, which in turn depends on the doping. If $a \gtrsim 20 \mu \mathrm{m}$, the spacing between CPA zeros, given in (8), becomes small, and the material index always passes close to one or more of them without fine-tuning $a$, making the behavior shown in Fig. 3 robust. Other indirect band gap semiconductors such as GaP show similarly good results.

The perfect absorption of a CPA arises from a combination of interference and dissipation: The reflected part of the first incident beam interferes destructively with the transmitted part of the second incident beam, and vice versa, and therefore the radiation is trapped in an interference pattern within the slab and lost entirely to dissipation. One sees from Fig. 3 that the other, orthogonal eigenmode has maximal scattering $\left(|s|^{2}\right)$. In fact, the energy absorbed in this mode is significantly less than if the system is incoherently illuminated from both sides; the reflected part of each beam interferes constructively with the transmitted part of the other beam, causing the radiation to escape the slab more quickly. Thus, a CPA allows resonant control of absorption, either an increase to nearly $100 \%$ or a reduction to $<1 \%$ for some resonators (see Fig. 4).

To illustrate the role of interference, we write down the transfer matrix, a $2 \times 2$ matrix with unit determinant:

$$
T(n, k)=\frac{1}{t}\left[\begin{array}{cc}
t^{2}-r^{2} & r \\
-r & 1
\end{array}\right]
$$

where $r(n, k)$ and $t(n, k)$ are the reflection and transmission amplitudes, respectively, for a single wave of unit amplitude incident from either direction. Perfect absorption occurs when $T_{11}=0$, i.e., when $r^{2}=t^{2}$. For the output beams to interfere destructively in the manner described above, not only must they have equal intensities $\left(|r|^{2}=\right.$ $|t|^{2}$ ), they must also have the correct relative phase. This should be impossible to satisfy when $n$ is real due to energy conservation, and the $T$-matrix analysis confirms this as follows. When $n$ is real, $T^{*}=T^{-1}$, and consequently (11) 


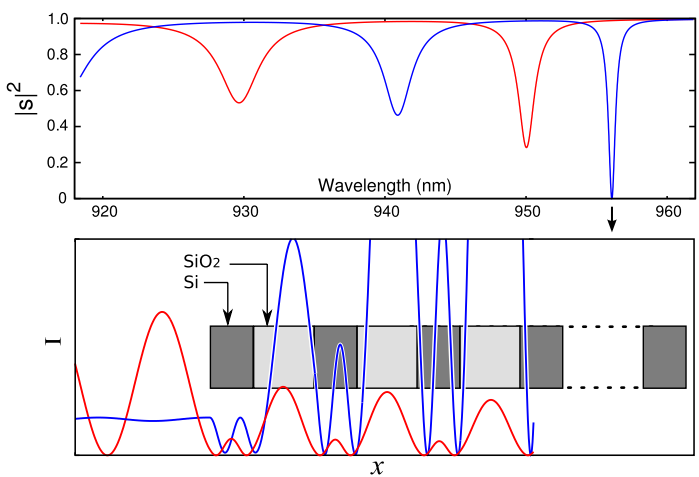

FIG. 4 (color online). Plot of output intensities $|s|^{2}$, as a function of wavelength, for a distributed feedback CPA consisting of $20 \mathrm{Si}$ slabs of width $188.5 \mathrm{~nm}$, separated by $\mathrm{SiO}_{2}$ slabs of width $266 \mathrm{~nm}$. The refractive index of $\mathrm{SiO}_{2}(n=1.46)$ is real to a good approximation in this wavelength range, so absorption occurs only in the Si. A large ( $99 \%$ ) absorption contrast can be observed at $956 \mathrm{~nm}$ near the photonic band gap. Inset: The intensity of the two eigenmodes, as a function of $x$, close to the edge of the system, at $956 \mathrm{~nm}$.

implies that, even if we achieve the condition $|r|^{2}=|t|^{2}$, there is always a $\pm \pi / 2$ phase difference between $r$ and $t$ and not the required $0, \pi$. This analysis is easily generalized to systems lacking mirror symmetry.

A very important property of the CPA which follows from this analysis is that we can change the output intensity simply by changing the relative phase of the input beams. For the parity symmetric two-channel CPA just discussed, a phase-modulated input $\left[1, \pm e^{i \phi}\right]$, with equal amplitude in each channel, leads to equal scattering intensities in each channel of the form

$$
I=I_{0} \sin ^{2}\left(\frac{\phi}{2}\right) .
$$

Here $I_{0}=\left|\left(n^{2}-n_{0}^{2}\right) /\left(n^{2}+n_{0}^{2}\right)\right|^{2}$, where $n$ is the special refractive index satisfying (7). In general, any CPA can function as a compact absorbing multichannel interferometer. Unlike an ordinary interferometer, a CPA does not shift the input beams between possible output channels but causes them to be absorbed within the material.

The absorbed photon energy may flow out of the cavity in a number of different forms. In direct band gap materials, a large fraction will be reemitted via fluorescence, which is not generally desirable, whereas in indirect band gap materials such as $\mathrm{Si}$, it can be extracted in the form of heat and/or photocurrent, either of which could be useful. Therefore, materials which are not useful as lasing media, such as $\mathrm{Si}$, make good CPAs, whereas good lasing materials, such as GaAs, make poor CPAs.

In applications it will often be useful to have a large contrast between the amount of scattered radiation which occurs at the CPA resonance condition versus incoherent or incorrectly phase-matched illumination. For the uniform CPA resonator described by (12), the intensity contrast is controlled by $n$, and for the Si CPA of Fig. 3, the maximal contrast is $\sim 0.75$. It is possible to increase the contrast substantially with a nonuniform system; e.g., a distributed feedback $\mathrm{Si} / \mathrm{SiO}_{2} \mathrm{CPA}$ of the type shown in Fig. 4 exhibits $\left|s_{1}\right|^{2} \sim 5 \times 10^{-4}$ for the absorbing eigenmode and $\left|s_{1}\right|^{2} \sim$ 0.99 for the other eigenmode. The absorption in the second mode is exceptionally low because the field inside the slab is concentrated in the $\mathrm{SiO}_{2}$ regions, which are nonabsorbing.

CPAs are potentially useful as transducers, modulators, or optical switches, for example, in on-chip integrated optical circuits based on Si waveguide or resonator technology [14]. We have verified that with realistic nonoptimized parameters a $\mathrm{Si}$ single-mode waveguide with $0.9 \mu \mathrm{m}$ distributed-Bragg-reflector mirrors and a $4 \mu \mathrm{m}$ "loss region" of pure intrinsic Si exhibits a CPA absorption resonance at $947 \mathrm{~nm}$ with contrast of roughly $90 \%$. Operation likely can be extended into the communications wavelengths around $1.5 \mu \mathrm{m}$ by designing devices with index tuning via free carrier injection as has already been achieved in other Si-based resonant photonic circuits [14]. However, solar photovoltaic or stealth applications appear unlikely, as CPAs are narrow-band devices and the oscillatory frequency dependence of their absorption tends to average out the response to a broadband signal.

This work was partially supported by NSF Grants No. DMR-0808937 and No. DMR-0908437, and by seed funding from the Yale NSF-MRSEC (DMR-0520495). We thank Hakan Türeci, Marin Soljačić, John Joannopoulos, Eric Ippen, Qinghai Song, Heeso Noh, and Michal Lipson for helpful discussions.

*yidong.chong@yale.edu

[1] H. Haken, Laser Light Dynamics (North Holland, Amsterdam, 1986).

[2] H. Cao, J. Phys. A 38, 10497 (2005).

[3] H. E. Türeci, L. Ge, S. Rotter, and A. D. Stone, Science 320, 643 (2008).

[4] K. M. Frahm et al., Europhys. Lett. 49, 48 (2000).

[5] A. E. Siegman, Phys. Rev. A 39, 1253 (1989).

[6] W. A. Hamel and J. P. Woerdman, Phys. Rev. A 40, 2785 (1989).

[7] P. L. Kapur and R. Peierls, Proc. R. Soc. A 166, 277 (1938).

[8] H. E. Türeci, A. D. Stone, and B. Collier, Phys. Rev. A 74, 043822 (2006).

[9] E. P. Wigner and L. Eisenbud, Phys. Rev. 72, 29 (1947).

[10] We choose the basis set corresponding to the dissipationless system, i.e., $n=n^{\prime}$, so that the imaginary part of the $R$ matrix is expressed explicitly in terms of $\operatorname{Im}\left\{n^{2}\right\}$.

[11] M. Cai, O. Painter, and K. J. Vahala, Phys. Rev. Lett. 85, 74 (2000).

[12] J. R. Tischler, M. S. Bradley, and V. Bulović, Opt. Lett. 31, 2045 (2006).

[13] S. Adachi, Optical Constants of Crystalline and Amorphous Semiconductors (Springer, New York, 1999).

[14] M. Lipson, J. Lightwave Technol. 23, 4222 (2005). 